\title{
Nefropatías y diuréticos
}

\author{
Nephropathies and diuretics \\ Leslie L. Santiago-Mejía ${ }^{a}$, Melina Carballo-Fajardo ${ }^{a^{*}}$, Karla A. Reyes-Minguez ${ }^{a}$
}

\begin{abstract}
:
Diuretics are substances that cause the elimination of water and electrolytes from the body through urine, increase urine fluid and secretion of solutes in it. The most common clinical use of diuretics is to reduce the volume of extracellular fluid. It has various classes: Osmotic, handles, antacids, carbonic anhydrase inhibitors, Aldosterone antagonists, sodium channel blockers.

Nephropathies (formerly nephrosis) is damage, disease or pathologies of the kidney. They consist of two categories: 1.-Acute renal injury. 2.-Chronic nephropathies. Acute renal injury is subdivided into: Prerenal, intrarenal and postrenal renal injury. Chronic nephropathy is caused by: Metabolic disorders. Hypertension, urinary tract infection or obstruction
\end{abstract}

\section{Keywords:}

Diuretics, nephropathies, nephrons, resorptions, cotransport, secretion, electrolytes

\section{Resumen:}

Los diuréticos son sustancias que provocan una eliminación de agua y electrolitos del organismo a través de la orina; aumentan el líquido de orina y secreción de solutos en esta. Uso clínico más común de los diuréticos es reducir el volumen de líquido extracelular. Tiene diversas clases: Osmóticos, de asas, tiacídos, inhibidores de la anhidrasa carbónica, antagonistas de la Aldosterona, bloqueantes de los canales de Sodio. Las nefropatías (anteriormente nefrosis) es el daño, enfermedad o patologías del riñón. Constan de dos categorías: 1.-Lesión renal aguda. 2.-Nefropatías crónicas. La lesión renal aguda se subdivide en: Lesión renal prerrenal, intrarrenal y postrrenal. La nefropatía crónica es causada por: Trastornos metabólicos, Hipertensión, Infección u obstrucción de vía urinaria.

\section{Palabras Clave:}

Diureticos, nefropanias, nefronas, reabsorcion,cotransporte, secrecion, electrolitos 
Publicación semestral, Tepexi Boletín Científico de la Escuela Superior Tepeji del Río, Vol. 7, No. 13 (2020) 30-31

\section{Referencias}

[1] Hall, J. E., \& Guyton, A. C. (2016). Guyton \& Hall. Tratado de fisiología médica (13a. ed.). Barcelona, España: Elsevier 